\title{
Inhibition of the growth and metastasis of human colon cancer by restoration of RUNX3 expression in cancer cells
}

\author{
ZHIHAI PENG ${ }^{1}$, HUAMEI TANG ${ }^{2}$, XIAOLIANG WANG ${ }^{1}$, CHONGZHI ZHOU ${ }^{1}$, JUNWEI FAN ${ }^{1}$, \\ LIWEI WANG ${ }^{3}$, ZHILIANG JIA ${ }^{4}$, QIANG LI ${ }^{4}$, XIANGDONG LE ${ }^{4}$, DAOYAN WEI ${ }^{4}$ and KEPING XIE ${ }^{4}$ \\ Departments of ${ }^{1}$ General Surgery, ${ }^{2}$ Pathology, ${ }^{3}$ Oncology, Shanghai Jiaotong University \\ Affiliated First People's Hospital, Shanghai, P.R. China; ${ }^{4}$ Department of Gastrointestinal Medical Oncology, \\ The University of Texas M.D. Anderson Cancer Center, Houston, TX, USA
}

Received March 24, 2008; Accepted May 23, 2008

DOI: 10.3892/ijo_00000085

\begin{abstract}
Recent studies demonstrated an epigenetic inactivation of the runt-related transcription factor 3 (RUNX3) gene in human colon cancer. However, it remains unclear whether RUNX3 is tumor suppressive in colon cancer and, if so, the underlying molecular mechanisms of this activity are still unknown. In the present study, we sought to determine the level of RUNX3 expression in human colon tumor specimens and used an animal model of colon cancer to determine the impact of RUNX3 expression on tumor growth and metastasis. First, we analyzed RUNX3 expression in 83 human colon tumor specimens using immunohistochemical, reverse transcriptasepolymerase chain reaction, and Western blot analysis. RUNX3 mRNA and protein expression levels were consistently lower in tumor tissue specimens than in matched normal colon tissue specimens. Also, restoration of RUNX3 expression in colon cancer cells using gene transfer inhibited colon tumor growth and metastasis in our animal model, which was consistent with inhibition of colon tumor growth in vitro. Collectively, our clinical and experimental data support the notion that RUNX3 is a tumor suppressor in human colon cancer.
\end{abstract}

\section{Introduction}

Colorectal cancer is one of the leading causes of cancer-related deaths worldwide (1). In China in particular, because of changes in lifestyle and diet, the incidence of colon cancer is increasing (2). Advances in the treatment of this disease are likely to come from a better understanding of its biology and behavior. In its early stages, colon carcinogenesis is associated with alterations in molecular signaling pathways; it then

Correspondence to: Dr Keping Xie, Department of Gastrointestinal Medical Oncology, Unit 426, The University of Texas M.D. Anderson Cancer Center, 1515 Holcombe Boulevard, Houston, TX 77030, USA

E-mail:kepxie@mdanderson.org

Key words: RUNX3, angiogenesis, metastasis, tumor, colon progresses as a result of a sequential accumulation of events that either activate oncogenes or inactivate tumor suppressor genes (3). These events affect the downstream signal transduction pathways that control cell growth and differentiation. Some alterations in multistep colon carcinogenesis affect $A P C$, $K$-Ras, p53, and elements of the transforming growth factor (TGF)- $\beta$ signaling pathway, such as TGF $\beta R I I$ and SMAD4. The TGF- $\beta$ signaling pathway serves as a major tumor suppressor in a variety of human gastrointestinal tumors, including colon cancer (4).

Recent studies indicated the role of the runt-related transcription factor 3 (RUNX3) gene in gastric and colorectal cancer development and progression $(5,6)$. The human $R U N X$ genes encode the $\alpha$ subunit of the Runt-domain transcription factor PEBP2/CBF (7) and are homologues of the Drosophila genes runt (8) and lozenge (9). The mammalian and Drosophila RUNX genes share an evolutionarily conserved region of 128 amino acids (the Runt domain) required for DNA binding and heterodimerization with PEBP2/CBF (7). All three Runtdomain family members, RUNX1, RUNX2 and RUNX3, are master regulators of gene expression in major developmental pathways and play pivotal roles in cell proliferation and differentiation in humans (10-12). Also, recent studies linked alterations of $R U N X$ genes with carcinogenesis (6,13-18). Furthermore, authors reported that a drastic loss of RUNX3 expression correlated with poor prognosis in patients with gastric cancer (19).

RUNX3 appears to be inactivated by several mechanisms, including promoter hypermethylation (silencing) and protein mislocalization in cancer cells $(19,20)$. However, whether and, if so, how RUNX3 regulates the growth and metastasis of colon cancer are unknown. Therefore, in the present study, we examined the expression of RUNX3 in colon tumor and matched normal colon tissue specimens obtained from patients with resected colon cancer. Loss of RUNX3 expression was lower in a subgroup of colon tumor specimens than in the matching subgroup of normal colon tissue specimens. Also, for the first time, we found that restoration of RUNX3 expression in colon cancer cells attenuated the growth and abrogated the metastasis of colon cancer in an animal model. These clinical and experimental data further substantiate RUNX3 as a tumor suppressor in human colon cancer. 


\section{Materials and methods}

Human tissue specimens. Human colon tumor specimens preserved in the Colon Cancer Tissue Bank at Shanghai Jiaotong University Affiliated First People's Hospital in Shanghai, P.R. China, were used in this study. Primary colon cancer in these patients was diagnosed and treated at the hospital from 2001 to 2003 . None of the patients underwent preoperative chemotherapy or radiation therapy. Eighty-three cases were selected to represent all of the stages and histological types of malignant colon cancer. All of the tissue specimens were obtained with the patients' informed consent, and all laboratory work involving human specimens was approved by the Institutional Review Board of and performed at Shanghai Jiaotong University Affiliated First People's Hospital.

Cell lines and culture conditions. The human colon cancer cell lines DLD-1, HCT-116, HT-29, RKO, SW480 and SW620, and the N87 human gastric cancer cell line were purchased from the American Type Culture Collection (Manassas, VA), and the colon cancer cell line Km12SM was provided by Isaiah J. Fidler (The University of Texas M.D. Anderson Cancer Center, Houston, TX). Both HT-29 and Km12SM, which have relatively low levels of RUNX3 expression, are tumorigenic in nude mice. The cell lines were maintained in plastic flasks as adherent monolayers in minimal essential medium supplemented with $10 \%$ fetal bovine serum, sodium pyruvate, nonessential amino acids, L-glutamine, and a vitamin solution (Flow Laboratories, Rockville, MD).

Restoration of RUNX3 expression in colon cancer cells. Recombinant adenoviruses harboring RUNX3 with a FLAG tag (Ad-RUNX3) or EGFP (Ad-EGFP) were generated using the AdEasy Adenoviral Vector System (Stratagene, La Jolla, CA). Replication-defective adenoviruses were expanded in HEK293 cells and purified using double $\mathrm{CsCl}$ gradient centrifugation to achieve a titer of $\sim 10^{10}$ plaque-forming units $/ \mathrm{ml}$. For adenoviral transduction, tumor cells were seeded in culture plates. Twelve hours later, the cells were incubated for $2 \mathrm{~h}$ at $37^{\circ} \mathrm{C}$ in serum-free medium alone or with AdRUNX3 or Ad-EGFP at a multiplicity of infection of 20 . After being washed with serum-free medium, the transduced cells were replenished with Dulbecco's modified Eagle's medium and incubated for indicated periods before harvesting of supernatants or cells for further use (19).

Western blot analysis. Whole-cell lysate extracts were extracted from human colon cancer cell cultures and tumor specimens. Standard Western blotting was performed using a rabbit polyclonal antibody against human RUNX3 at a dilution of 1:200 (Active Motif, Carlsbad, CA) or FLAG (M2; Sigma-Aldrich, St. Louis, MO) and the anti-rabbit IgG antibody, a horseradish peroxidase-linked $\mathrm{F}\left(\mathrm{ab}^{\prime}\right)_{2}$ fragment obtained from a donkey (Amersham, Arlington Heights, IL). Equal protein sample loading was monitored by probing the same membrane filter with an anti- $\beta$-actin antibody (19). The probe proteins were detected using the Amersham enhanced chemiluminescence system according to the manufacturer's instructions.
Animals. Female athymic nude mice were purchased from The Jackson Laboratory (Bar Harbor, ME). The mice were housed in laminar flow cabinets under specific pathogen-free conditions and used when they were 8 weeks old. The animals were housed in facilities approved by the Association for Assessment and Accreditation of Laboratory Animal Care in accordance with the current regulations and standards of the U.S. Department of Agriculture, Department of Health and Human Services, and National Institutes of Health. All experiments involving use of animals were performed at the M.D. Anderson Cancer Center with approval from its Institutional Animal Care and Use Committee.

Tumor growth and metastasis. To prepare colon tumor cells for inoculation in the mice, cells in the exponential growth phase were harvested by brief exposure to a $0.25 \%$ trypsin/ $0.02 \%$ ethylenediaminetetraacetic acid solution (wt/vol). Cell viability was determined using trypan blue exclusion, and only single-cell suspensions that were $>95 \%$ viable were used. Tumor cells $\left(1 \times 10^{6}\right.$ cells/mouse) were then injected into the subcapsule of the cecum in nude mice in groups of 10 . The animals were sacrificed 60 days after the tumor-cell injection or when they had become moribund, and their primary colon tumors were harvested and weighed. In addition, each mouse liver was removed and fixed in Bouin solution for $24 \mathrm{~h}$ to differentiate the neoplastic lesions from the organ parenchyma; metastases on the surface of the liver were counted (double-blinded) under a dissecting microscope.

Immunohistochemical analysis of colon tumor and normal colon tissue specimens. Sections $(5 \mu \mathrm{m})$ of formalin-fixed, paraffin-embedded colon tumor specimens were prepared and processed as described previously (21). RUNX3 protein expression in the sections was detected with an anti-RUNX3 antibody. A RUNX3-positive reaction was indicated by a reddish-brown precipitate in the nucleus and cytoplasm. Two independent investigators scored the sections without knowledge of the patient outcome (double-blinded). Mean values of two independent scores were calculated in our study. Depending on the percentage of RUNX3-positive cells and staining intensity, RUNX3 staining positivity was classified according to three groups: negative/weakly positive, moderately positive, and strongly positive $(19,21)$.

Statistics. The two-tailed $\chi^{2}$ test was performed to determine the significance of the relationships among the covariates. Each experiment was performed independently at least twice with similar results; one representative experiment was presented. The significance of the in vitro data was determined using the Student's t-test (two-tailed), whereas that of the in vivo data was determined using the two-tailed Mann-Whitney $\mathrm{U}$ test. $\mathrm{P}$ values $<0.05$ were deemed significant.

\section{Results}

Patient and specimen characteristics. The patients consisted of $40(48 \%)$ men and 43 (52\%) women. Eighteen (22\%) patients were $>60$ years old, and $65(78 \%)$ were $\leq 60$ years old; the mean age was 65.1 years. The primary tumor was located in the rectum in $28(34 \%)$ patients, the sigmoid colon in 17 

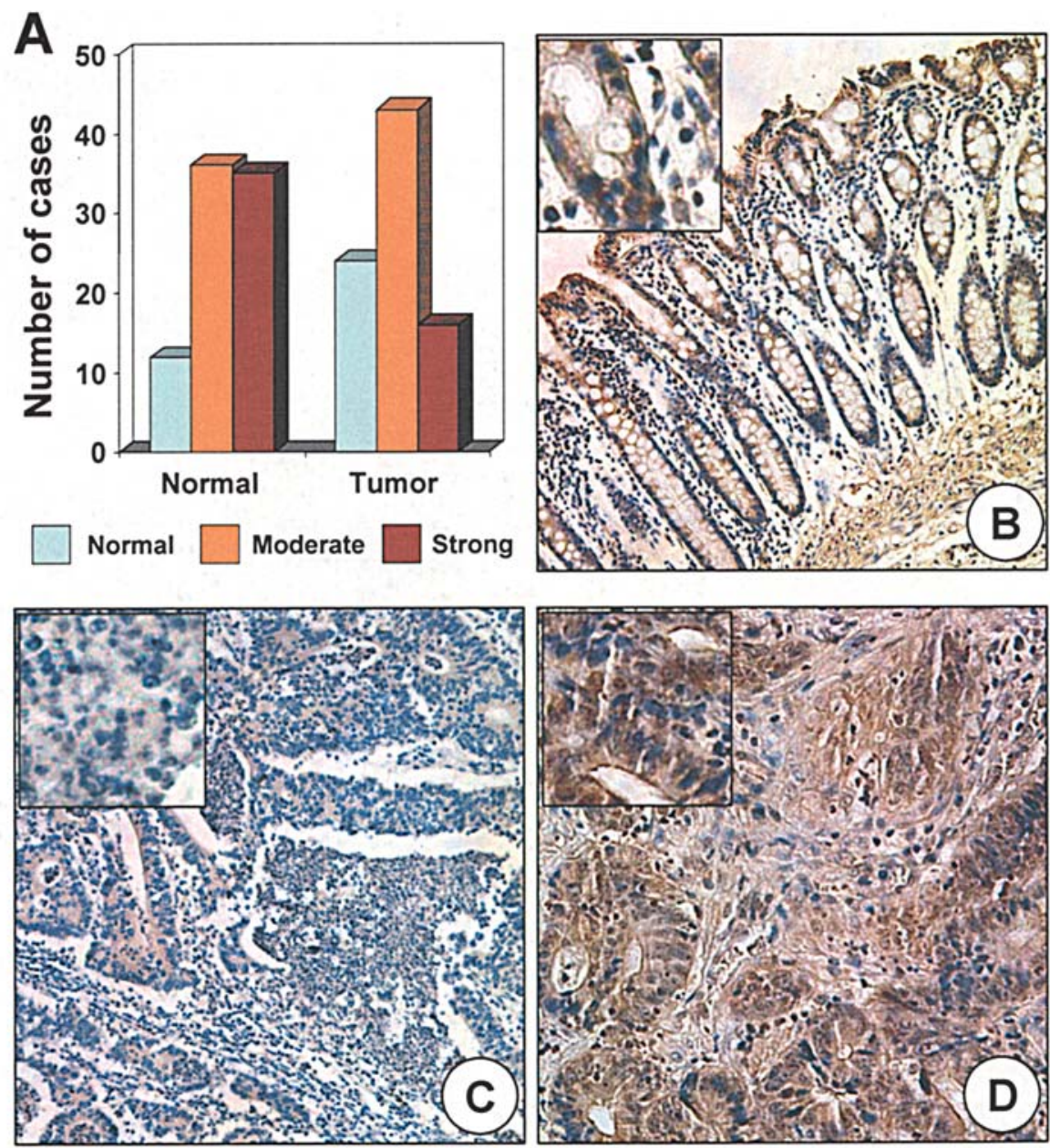

Figure 1. Immunostaining for RUNX3 protein. Sections of formalin-fixed, paraffin-embedded human colon tumor specimens (83 cases) were prepared. Immunohistochemical staining of the sections was performed using specific antibodies against RUNX3. (A) RUNX3 expression levels were significantly lower in tumor specimens than in matched normal colon tissue specimens (Pearson's $\chi^{2}$ test, $\left.\mathrm{P}<0.001\right)$. Shown are photos of $(\mathrm{B})$ strongly RUNX3-positive immunostaining and (C) RUNX3-negative immunostaining in tumor tissue and (D) strongly RUNX3-positive immunostaining in normal colon tissue (original magnification, $\mathrm{x} 400$ for the inserts, $\mathrm{x} 100$ for all other images).

(20\%) patients, the ascending colon in $27(33 \%)$ patients, the descending colon in $5(6 \%)$ patients, and the transverse colon in $6(7 \%)$ patients. Seventeen $(20 \%)$ of the tumors were well differentiated, $43(52 \%)$ were moderately differentiated, and $23(28 \%)$ were poorly differentiated. Twelve (14\%) were grade I tumors, 46 (55\%) were grade II tumors, and $25(30 \%)$ were grade III tumors. Forty-two $(51 \%)$ of the cases were node-positive, whereas $41(49 \%)$ were node-negative. Finally, $63(76 \%)$ cases were negative for distant metastasis, whereas $20(24 \%)$ were positive for distant metastasis.

Loss of RUNX3 expression in subsets of colon tumor and normal colon tissue specimens. We first determined the levels of RUNX3 expression in the primary colon tumor and matched normal colon tissue specimens obtained from the 83 patients. Twelve (14\%), 36 (43\%), and $35(42 \%)$ of the normal tissue specimens had negative/weak, moderate, and strong RUNX3 immunostaining, respectively. In comparison, 24 (29\%), $43(52 \%)$, and $16(19 \%)$ of the tumor specimens had weak/negative, moderate, and strong RUNX3 immunostaining, respectively. The levels of RUNX3 expression were significantly lower in the colon tumor specimens than in the normal colon tissue specimens (Pearson's $\chi^{2}$ test, $\mathrm{P}=0.003$ ) (Fig. 1).

To confirm these immunostaining findings, we analyzed sets of colon tumor and matched normal colon tissue specimens for RUNX3 mRNA expression. We selected 14 cases with intact RNA integrity for reverse transcriptase (RT)-polymerase chain reaction (PCR) analysis. Five of these cases (36\%) exhibited significantly lower RUNX3 mRNA expression in the colon tumor specimens than in the respective normal colon tissue specimens (Fig. 2). We then selected the cases with substantially (cases 5, 6, 9, 13 and 15) or marginally (cases 14, 16 and 17) lower RUNX3 mRNA expression in colon tumor specimens for RUNX3 protein expression analysis using Western blotting. In general, the results of this Western blot analysis were consistent with those of the RT-PCR analysis (Fig. 3). However, the Western blot analysis appeared to show more cases with lower RUNX3 expression in colon tumor specimens, which was consistent with the immunostaining results. Therefore, combined use of immunostaining and Western blotting was the most reliable in determining the RUNX3 expression status in the specimens. 

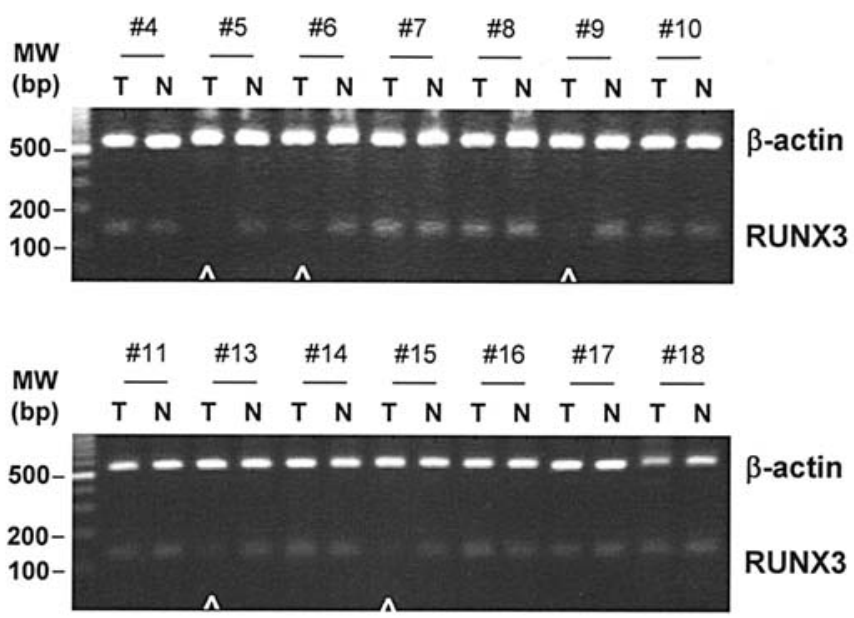

Figure 2. RT-PCR analysis of RUNX3 mRNA expression. Total RNA was extracted from colon tumor and normal colon tissue specimens, and the integrity of RNA in the specimens was verified using RT-PCR with specific primers for $B$-actin. Both RUNX3 and B-actin were then amplified using RT-PCR with primers for both genes. This was a representative experiment of three with similar results. Substantially lower RUNX3 mRNA expression in the colon tumor specimen than in the matched normal colon tissue specimen.
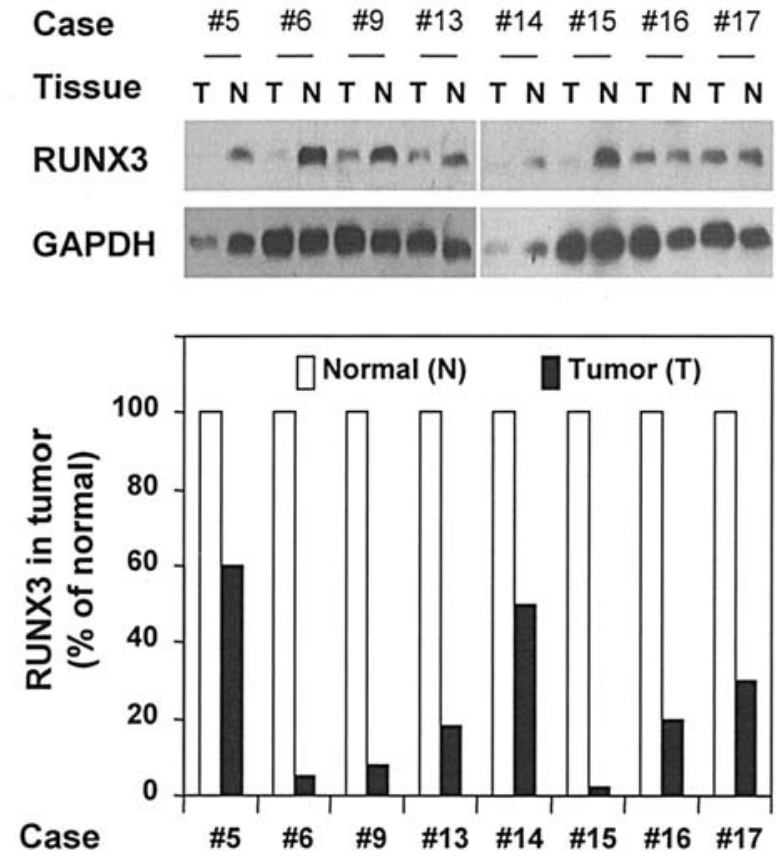

Figure 3. Western blot analysis of RUNX3 protein expression. Total cellular protein was extracted from colon tumor and normal colon tissue specimens. Both RUNX3 and B-actin were then determined using specific antibodies against RUNX3 and $B$-actin via Western blot analysis. This was a representative experiment of three with similar results. Substantially lower RUNX3 mRNA expression in the colon tumor specimen than in the normal colon tissue specimen.

Restoration of RUNX3 expression in human colon cancer cells. To provide causal evidence of the role of RUNX3 in colon cancer development and progression, we first determined the level of RUNX3 protein expression in various human colon cancer cell lines using the cell lysate of N87
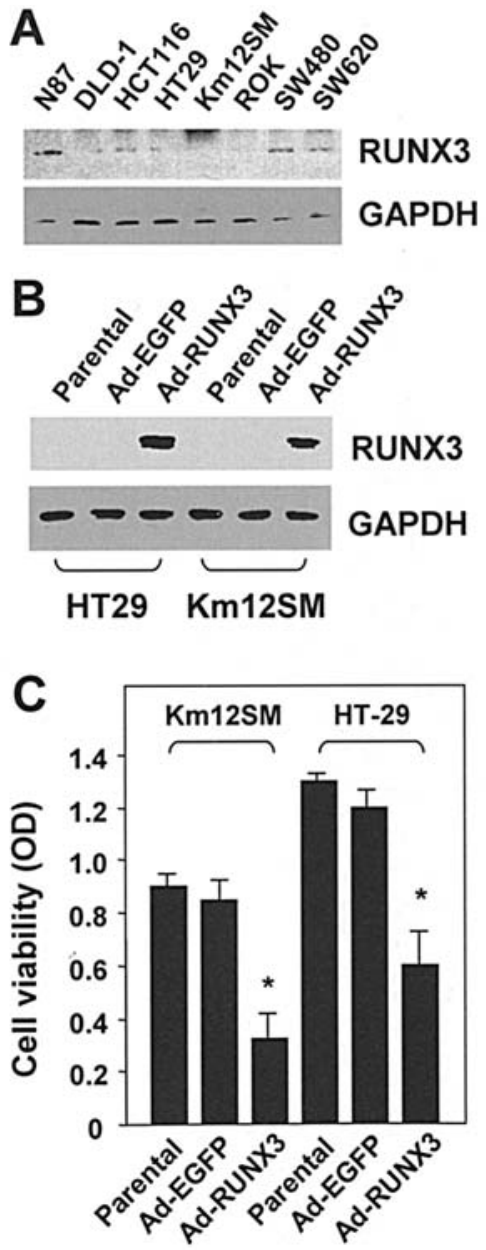

Figure 4. Restoration of RUNX3 expression and suppression of colon tumor growth in vitro. (A) RUNX3 protein expression levels were determined in various human colon cancer cell lines using Western blot analysis. GAPDH, glyceraldehyde-3-phosphate dehydrogenase. (B) HT-29 and Km12SM cells transduced with control Ad-EGFP or Ad-RUNX3 at a multiplicity of infection of 20 were incubated for $24 \mathrm{~h}$. Untreated cells were used as controls (Parental). Total protein lysates were harvested from cell cultures, and the RUNX3 expression levels in tumor cells were determined using Western blot analysis with an anti-FLAG antibody. (C) HT-29 and Km12SM cells were seeded into six-well plates in triplicate with a RUNX3 expression vector or control vector and incubated for $24 \mathrm{~h}$. The cell viability was determined $48 \mathrm{~h}$ after transduction. This was a representative experiment of three with similar results. ${ }^{*} \mathrm{P}<0.01$ as compared with respective control groups.

human gastric cancer cells as a positive control as previously reported (19). We detected various levels of RUNX3 protein expression in these cell lines. Interestingly, we observed lower RUNX3 expression in SW620, which was originally established from a liver metastasis, than in SW480, which was originally established from the primary tumor in the same patient from whom SW620 was established (Fig. 4A).

Inhibition of colon cancer growth in vitro by restoration of RUNX3 expression. Next, we transduced HT-29 and Km12SM, which have low to nondetectable levels of RUNX3 expression, with Ad-RUNX3. Using Western blot analysis, we observed that exogenous RUNX3 was expressed in tumor cells transduced with Ad-RUNX3 but not in untreated control tumor cells or tumor cells transduced with control Ad-EGFP 
A
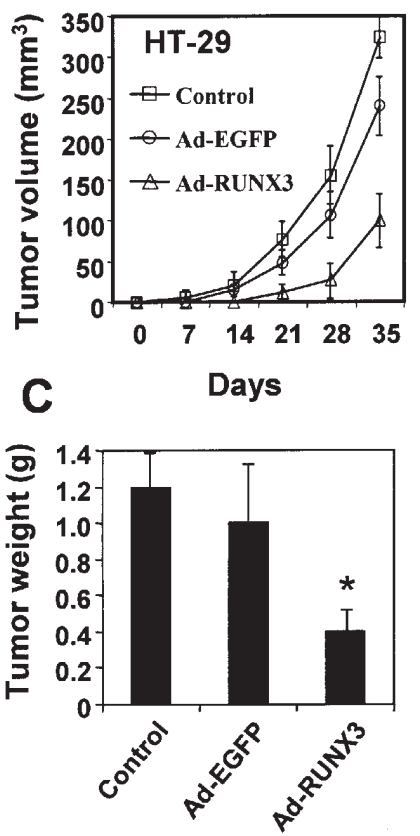

Figure 5. RUNX3 inhibited human colon colon tumor growth and metastasis in a nude mouse model. (A) HT-29 and (B) Km12SM cells transduced with control Ad-EGFP or Ad-RUNX3 (1x10 $10^{6}$ cells/mouse) were injected into the subcutis of nude mice $(n=5)$. Untreated cells were used as controls. The resulting tumors were measured once a week. This was one representative experiment of two with similar results. Km12SM cells transduced with control Ad-EGFP or Ad-RUNX3 ( $1 \times 10^{6}$ cells/mouse) were injected into the cecum in nude mice $(n=5)$. The animals were sacrificed 35 days after the tumor-cell injection or when they had become moribund. (C) Tumors were weighted, and (D) metastases to regional lymph nodes and the liver were evaluated. This was one representative experiment of two with similar results. ${ }^{*} \mathrm{P}<0.01$ as compared with respective control groups.

(Fig. 4B). To determine the impact of this restored RUNX3 expression on the growth of human colon cancer cells, we incubated control HT-29 and Km12SM cells, HT-29 and Km12SM cells transduced with Ad-RUNX3, and HT-29 and Km12SM cells transduced with Ad-EGFP for $48 \mathrm{~h}$ after gene transfer. We determined viable cells using an MTT assay. Restored RUNX3 expression in colon cancer cells clearly inhibited the growth of both HT-29 and Km12SM cells in vitro (Fig. 4C), showing that RUNX3 has a tumor-suppressive function in human colon cancer cells.

Suppression of colon tumor growth and abrogation of metastasis by RUNX3. To determine the effect of RUNX3 on colon tumor growth kinetics, we subcutaneously injected HT-29 and Km12SM cells into nude mice. Untreated control tumor cells and tumor cells transduced with Ad-EGFP produced progressively growing tumors, whereas RUNX3transduced tumor cells only produced slow-growing tumors (Fig. 5A and B). To be more biologically relevant, we injected $\mathrm{Km} 12 \mathrm{SM}$ cells into the cecal wall in mice in groups of five (orthotopic gastric cancer animal model). Control and AdEGFP-transduced tumor cells produced large tumors that metastasized to regional lymph nodes and the liver, whereas RUNX3-transduced tumor cells only produced localized small tumors (Fig. 5C and D). Therefore, enforced RUNX3 expression suppressed human colon cancer growth and metastasis.

\section{Discussion}

Although researchers have implicated RUNX3 as a tumor suppressor in several malignancies, including gastric cancer $(6,19,22,23)$, the impact of RUNX3 alterations on colon cancer has gone unexamined. In the present study, we offered the first clinical and experimental evidence that altered expression of RUNX3 in tumor cells directly suppresses colon cancer growth and metastasis. Specifically, we found the loss of RUNX3 protein expression in human colon cancer cells. Restoration of RUNX3 expression in these cells significantly inhibited colon cancer cell growth in vitro and tumorigenicity and metastasis in an animal model.

Studies have identified a causal relationship between the loss of RUNX3 expression and the incidence of gastric cancer in humans as well as mice $(6,19)$. Goel and colleagues elegantly demonstrated that the majority of human colon cancer cell lines do not express RUNX3 and that this lack of expression is significantly attributed to hypermethylation of the promoter region of the gene (5). Notably, the RUNX3 promoter is methylated in colorectal tumors but not in adjacent nonmalignant mucosal epithelium, suggesting that methylation of this promoter is a cancer-specific event. Consistently, in the present study, we clearly showed that expression of RUNX3 mRNA and protein was substantially downregulated in human colon cancer cells and tumor specimens. Our observation that RUNX3 expression is frequently silenced in colon cancer cell lines and primary colorectal tumors is consistent with the theory that RUNX3, localized to a strategic location on $1 \mathrm{p} 36$, is likely a significant candidate tumor suppressor gene previously not shown to play a role in the development of colorectal cancer. This is strongly supported by our findings showing that engineered restoration of RUNX3 expression in colon cancer cells suppressed the growth and metastasis of human colon cancer cells. Collectively, these findings provide direct evidence that RUNX3 functions as a tumor suppressor in human colon cancer,

Previous studies showed that the gastric epithelium in $R U N X 3^{-/}$knockout mice exhibits a reduced rate of apoptosis and reduced sensitivity to TGF- $\beta 1$, suggesting that the tumor suppressor activity of RUNX3 operates downstream of the TGF- $\beta$ signaling pathway $(6,22,23)$. Given the potential role of RUNX3 in TGF- $\beta$ signaling, the tumor suppressor activity of RUNX3 may be realized by inducing cell cycle arrest and/ or apoptosis. Indeed, the functions of RUNX proteins appear to be similar to those of members of the TGF- $\beta$ superfamily of cytokines (6). Presumably, TGF- $\beta$ and RUNX proteins cooperatively regulate cell growth and differentiation through multiple mechanisms. Several studies have shown physical interaction of RUNX protein with Smads and p300, suggesting the foundation of the functional cooperation between TGF- $\beta$ and RUNX proteins (24-27). Since TGF-ß generally induces cell cycle arrest at the G0/G1 phase by increasing the expression and/or activity of specific cyclin-dependent kinase inhibitors $(28,29)$ and many other potential targets $(30-33)$ and since cyclin D1 is an important positive cell cycle regulator whereas p27 is an important cell cycle inhibitor (34), 
whether they are actually involved in the regulation of cell cycle arrest and/or apoptosis by RUNX3 is currently under investigation in our laboratory.

In summary, our data indicate that $R U N X 3$ expression is clearly lost in a significant subgroup of human colon tumor specimens. Although future studies are needed to reveal the molecular basis of $R U N X 3$ function in colon physiology and colon cancer pathogenesis, we provided strong evidence that RUNX3 is a candidate tumor suppressor in colon carcinogenesis. Therefore, a better understanding of the molecular basis for the molecular function of the aberrant RUNX3 signaling pathway may help in designing effective therapies to control colon cancer growth and metastasis.

\section{Acknowledgements}

We thank Gerard Quinones for assistance in the preparation of the manuscript and Don Norwood for editorial comments. This work was supported, in part, by the Natural Science Foundation of the People's Republic of China (to Z.P.) and by Research Scholar Grant CSM-106640 from the American Cancer Society and Grant 1R01-CA093829 from the National Cancer Institute, National Institutes of Health (to K.X.).

\section{References}

1. Parkin DM, Bray FJ, Ferlay and Pisani P: Global cancer statistics, 2002. CA Cancer J Clin 55: 74-108, 2005.

2. Li M and Gu J: Changing patterns of colorectal cancer in China over a period of 20 years. World J Gastroenterol 11: 4685-4688, 2005.

3. Fearon ER and Vogelstein BA: Genetic model for colorectal tumorigenesis. Cell 61: 759-767, 1990.

4. Markowitz SD and Roberts AB: Tumor suppressor activity of the TGF- $\beta$ pathway in human cancers. Cytokine Growth Factor Rev 7: 93-102, 1996.

5. Goel A, Arnold CN, Tassone P, et al: Epigenetic inactivation of RUNX3 in microsatellite unstable sporadic colon cancers. Int J Cancer 112: 754-759, 2004.

6. Li QL, Ito K, Sakakura C, et al: Causal relationship between the loss of RUNX3 expression and gastric cancer. Cell 109: 113-124, 2002.

7. Ito Y: Molecular basis of tissue-specific gene expression mediated by the runt domain transcription factor PEBP2/CBF. Genes Cells 4: 685-686, 1999.

8. Kania MA, Bonner AS, Duffy JB and Gergen JP: The Drosophila segmentation gene runt encodes a novel nuclear regulatory protein that is also expressed in the developing nervous system. Genes Dev 4: 1701-1713, 1990.

9. Daga A, Karlovich CA, Dumstrei K and Banerjee U: Patterning of cells in the Drosophila eye by Lozenge, which shares homologous domains with AML1. Genes Dev 10: 1194-1205, 1996.

10. Karsenty G and Wagner EF: Reaching a genetic and molecular understanding of skeletal development. Dev Cell 2: 389-406, 2002.

11. Tracey WD and Speck NA: Potential roles for RUNX1 and its orthologs in determining hematopoietic cell fate. Semin Cell Dev Biol 11: 337-342, 2000.

12. Yang $\mathrm{S}$, Wei $\mathrm{D}$, Wang $\mathrm{D}$, et al: In vitro and in vivo synergistic interactions between the Runx2/Cbfa1 transcription factor and bone morphogenetic protein-2 in stimulating osteoblast differentiation. J Bone Miner Res 18: 705-715, 2003.
13. Westendorf JJ and Hiebert SW: Mammalian runt-domain protein and their roles in hematopoiesis, osteogenesis, and leukemia. J Cell Biochem (Suppl 32-33): 51-58, 1999.

14. Ito Y: Oncogenic potential of the RUNX gene family: 'overview' Oncogene 23: 4198-4208, 2004.

15. Levanon D, Brenner O, Otto F and Groner Y: Runx3 knockouts and stomach cancer. EMBO Rep 4: 560-564, 2003.

16. Levanon D, Bettoun D, Harris-Cerruti C, et al: The Runx3 transcription factor regulates development and survival of TrkC dorsal root ganglia neurons. EMBO J 21: 3454-3463, 2002.

17. Stewart M, MacKay N, Cameron ER and Neil JC: The common retroviral insertion locus Dsi1 maps 30 kilobases upstream of the P1 promoter of the murine Runx3/Cbfa3/Aml2 gene. J Virol 76: 4364-4369, 2002.

18. Kim R, Trubetskoy A, Suzuki T, et al: Genome-based identification of cancer genes by proviral tagging in mouse retrovirus-induced T-cell lymphomas. J Virol 77: 2056-2062, 2003.

19. Wei D, Gong W, Oh SC, et al: Loss of RUNX3 expression significantly affects the clinical outcome of gastric cancer patients and its restoration causes drastic suppression of tumor growth and metastasis. Cancer Res 65: 4809-4816, 2005.

20. Ito K, Liu Q, Salto-Tellez M, et al: RUNX3, a novel tumor suppressor, is frequently inactivated in gastric cancer by protein mislocalization. Cancer Res 65: 7743-7750, 2005.

21. Wang L, Wei D, Huang S, et al: Transcription factor Sp1 expression is a significant predictor of survival in human gastric cancer. Clin Cancer Res 9: 6371-6380, 2003.

22. Oshimo Y, Oue N, Mitani Y, et al: Frequent loss of RUNX3 expression by promoter hypermethylation in gastric carcinoma. Pathobiology 71: 137-143, 2004.

23. Kim TY, Lee HJ, Hwang KS, et al: Methylation of RUNX3 in various types of human cancers and premalignant stages of gastric carcinoma. Lab Invest 84: 479-484, 2004

24. Miyazono K, Maeda S and Imamura T: Coordinate regulation of cell growth and differentiation by TGF-beta superfamily and Runx proteins. Oncogene 23: 4232-4237, 2004.

25. Kitabayashi I, Yokoyama A, Shimizu K and Ohki M: Interaction and functional cooperation of the leukemia-associated factors AML1 and p300 in myeloid cell differentiation. EMBO J 17: 2994-3004, 1998.

26. Lee KS, Kim HJ, Li QL, et al: Runx2 is a common target of transforming growth factor beta1 and bone morphogenetic protein 2, and cooperation between Runx 2 and Smad5 induces osteoblast-specific gene expression in the pluripotent mesenchymal precursor cell line C2C12. Mol Cell Biol 20: 8783-8792, 2000

27. Zhang YW, Yasui N, Ito K, et al: A RUNX2/PEBP2alpha A/ CBFA1 mutation displaying impaired transactivation and Smad interaction in cleidocranial dysplasia. Proc Natl Acad Sci USA 97: 10549-10554, 2000

28. Weinberg RA: The retinoblastoma protein and cell cycle control. Cell 81: 323-330, 1995.

29. Sherr CJ: Cancer cell cycles. Science 274: 1672-1677, 1996.

30. Mithani SK, Balch GC, Shiou SR, et al: Smad3 has a critical role in TGF-beta-mediated growth inhibition and apoptosis in colonic epithelial cells. J Surg Res 117: 296-305, 2004.

31. Yamamura Y, Hua X, Bergelson S and Lodish HF: Critical role of Smads and AP-1 complex in transforming growth factorbeta-dependent apoptosis. J Biol Chem 275: 36295-36302, 2000.

32. Schuster N and Krieglstein K: Mechanisms of TGF-betamediated apoptosis. Cell Tissue Res 307: 1-14, 2002.

33. Chipuk JE, Bhat M, Hsing AY, Ma J and Danielpour D: Bcl-xL blocks transforming growth factor-beta 1-induced apoptosis by inhibiting cytochrome $\mathrm{c}$ release and not by directly antagonizing Apaf-1-dependent caspase activation in prostate epithelial cells. J Biol Chem 276: 26614-26621, 2001.

34. Malumbres $\mathrm{M}$ and Barbacid $\mathrm{M}$ : To cycle or not to cycle: a critical decision in cancer. Nat Rev Cancer 1: 222-231, 2001. 